\title{
BACK TO THE QUESTION OF ONTOLOGY (AND METAPHYSICS) ${ }^{1}$
}

\author{
JONAS RAFAEL BECKER ARENHART ${ }^{1}$ \\ https://orcid.org/0000-0001-8570-7336 \\ ${ }^{1}$ Universidade Federal de Santa Catarina \\ Department of Philosophy \\ Florianópolis, S.C. \\ Brazil \\ Universidade Federal do Maranbão \\ Graduate Program in Philosophy \\ São Luís, M.A. \\ Brazil \\ jonas.becker2@gmail.com
}

\author{
RAONI WOHNRATH ARROYO ${ }^{2}$ \\ https:// orcid.org/0000-0002-3800-8505 \\ ${ }^{2}$ Universidade Federal de Santa Catarina \\ Graduate Program in Philosophy \\ Florianópolis, S.C. \\ Brazil \\ raoniarroyo@gmail.com
}

\section{Article info \\ CDD: 110 \\ Keywords \\ Ontology \\ Metaphysics \\ Metaontology}

Received: 06.09.2020; Revised: 14.04.2021; Accepted: 04.05.2021

https://doi.org/10.1590/0100-6045.2021.V44N2.JR

1 This is a fully collaborative work. Author's names appear in alphabetical order. Jonas R. Becker Arenhart is partially supported by CNPq. Part of the research for this paper was developed while Jonas held a Capes-Humboldt Experienced Researcher Fellowship, at the Ruhr-University Bochum, Germany.

Manuscrito - Rev. Int. Fil. Campinas, v. 44, n. 2, pp. 01-51, Apr.-Jun. 2021. 
Metametaphysics

Naturalism

Abstract: We articulate a distinction between ontology, understood as involving existence questions, and metaphysics, understood as either providing for metaphysical profiles of entities or else as dealing with fundamentality and/or grounding and dependence questions. The distinction, we argue, allows a better understanding of the roles of metaontology and metametaphysics when it comes to discussing the relations between ontology and science on the one hand, and metaphysics and science on the other. We argue that while ontology, as understood in this paper, may have reasonable perspectives for naturalization, given its relation to science, the same cannot be said for metaphysics, given that it is typically understood as an additional theoretical layer over science, not participating in the scientific investigation. That may result either in skepticism over metaphysics, or else on accepting that metaphysics is an autonomous branch of investigation, depending on one's concern for metaphysics.

\section{Introduction}

Following the lead of Quine, ontology is traditionally said to deal with the question of what exists, while metaphysics, perhaps following Aristotle, works on a more shady area, dealing with some disparate issues such as being qua being, causality, theology, and in more recent times, the nature of modality, personal identity, free will, the nature of mind, among other things. Drawing such a division in the subject matter seems to indicate that they are indeed distinct areas of research. But are they? The question is important, because the methods employed to deal with problems in one area (questions of existence) may not fit profitably into the proper inquiry in the other area (more general metaphysical questions); the goals of one area may not be the goals of the other, and vice versa. In other words: debates in 
metaontology and metametaphysics also depend on how one sees the goals in each of the areas and also their relationship. In particular, given that metaontology and metametaphysics may be seen as specially concerned with epistemological issues related to ontology and metaphysics, respectively, this question of the appropriate delimitation of each area may be of special interest for those concerned with the epistemology for each of them.

To exemplify the importance of having some distinction between those fields, consider debates on whether some disputes in metaphysics/ontology are merely verbal, or perhaps the discussion concerning the relation between metaphysics/ontology and science, which is the one we shall be more concerned with. Only once ontology and metaphysics are properly understood, those issues, which involve higher-order claims, will be able to be properly addressed. Ontology, on the characterization we are adopting here, deals directly with existence questions and (typically with) the role of quantifiers, while metaontology discusses mostly whether existential quantifiers are up to the task of expressing existence and how to make objective existential claims; metaphysics, on the other hand, does not completely surrender to discussions on quantifiers and existence. In fact, there are many problems in metaphysics that simply cannot be dealt with by solely focusing on existence and quantifiers; the corresponding metametaphysical investigation will not be adequate if it is concerned only with the role of quantifiers in such investigations. So, identifying the two areas may lead to confusion also on the second-order investigation of methodology and goals of each area.

However, despite seeming a pretty natural and fruitful distinction, the separation between ontology and metaphysics is not always as clear as it should be, and this happens mostly where the distinction is of utmost 
importance: in discussions concerning the methodology of these areas. Perhaps it is not completely off the mark to say that part of the current identification of ontology and metaphysics lies in the offspring of the Carnap-Quine debate on ontology. While Carnap and Quine were-discussing ontology in the sense delineated above, and not metaphysics in a broader sense, descendants of Quine seem to be thinking of him as laying the grounds for the work in metaphysics in a much broader sense. Also, while Carnap was thinking about ontology when he proposed his approach to existence questions in "Empiricism, Semantics, and Ontology" (ESO), his descendants (the neo-Carnapians) apply this same approach overall, to many issues that do not seem to concern only problems of existence. Proposals concerned with ontology were extrapolated to metaphysics, with the result that confusions in the respective discussions on the methodology and epistemology of ontology and metaphysics have increased.

In this paper, we shall argue that keeping both areas separated brings benefits for current debates, in particular for the debate on the relation between ontology and science and the one hand, and metaphysics and science on the other. We are certainly aware that focusing on what seems to be terminological issues may lead to pseudo-problems, but we believe that the distinction we shall advocate here is full of consequences for the debate on the relation between ontology and metaphysics with science, a debate that occupies the center of the stage nowadays. That is, the distinction being advanced here, although quite general, bears important fruits for the relation of ontology and metaphysics with science, and this is the major concern that lies in the background of our discussion. That being said, in sections 2 and 3, we begin by distinguishing the areas in clearer terms, illustrating how ontology may be understood as the task of providing for a catalog of reality, and how 
metaphysics builds from there. In section 3, in particular, we suggest how the relation between ontology and metaphysics may be understood, once the distinction is drawn. That relation will allow one to keep both investigations separated, and distinct verdicts on the fruitfulness of establishing relations between ontology and science on the one hand, and metaphysics and science on the other, will emerge. In section 4, we argue that on a reasonable interpretation, the CarnapQuine debate on ontology indicates that there is a pragmatic approach to ontology that benefits, even if little, from the epistemic authority of science. The same cannot be clearly said of metaphysics. This indicates that a kind of naturalism may be, at least in principle, vindicated for ontological issues, but not so clearly for metaphysical issues. As a result, the distinction between ontology and metaphysics contributes to the epistemology of both areas, even if pointing negatively to the possible benefits that metaphysics may somehow derive from science. We conclude in section 5 .

\section{Ontology and metaontology}

Let us begin by putting in clear terms what is to be understood as the proper subject of ontology. While this cannot be done in a crystal-clear fashion for most academic disciplines (not even for mathematics and physics), some clarifying hints may be advanced (as it is typically done for mathematics and physics). That will also work for ontology, and for our purposes it will be enough to make it clear that, for good reasons, it should be understood as a field of research that is distinct from metaphysics. The idea here is not new, although the approach advanced may present some connections that will allow us also to illuminate second-order discussions. Basically, we conceive ontology as in the Quinean (and Carnapian) tradition, dealing with questions of 
existence (e.g., are there numbers? are there material bodies? and so on). The following is a nice summary of the proposed distinction, by Hofweber (the same distinction, but with different terminology, was already present in Arenhart 2012):

In metaphysics we want to find out what reality is like in a general way. One part of this will be to find out what the things or the stuff are that are part of reality. Another part of metaphysics will be to find out what these things, or this stuff, are like in general ways. Ontology, on this quite standard approach to metaphysics, is the first part of this project, i.e. it is the part of metaphysics that tries to find out what things make up reality. Other parts of metaphysics build on ontology and go beyond it, but ontology is central to it, [...]. Ontology is generally carried out by asking questions about what there is or what exists. (Hofweber 2016, p.13)

On this conception, ontology is a proper part of metaphysics, and it is concerned with the furniture of the world. One should attempt to make a list of the existing entities, in general terms. Although the ontological list is an important part of ontology, it does not fully exhaust what ontology is. Another part of ontology would consist in the classification of the entities obtained from the list into kinds, or types: are they particular objects, processes, events, properties, relations, or something else? Note that this does not involve yet doing metaphysics properly. Metaphysics will build on that list, trying to describe more generally the nature of those items and their metaphysical relations (dependence relations, questions of priority, and so on; we shall return to this in the next section). There is a further virtue of this 
approach in confining ontology to this specific task: it allows one to concentrate only on ontology, if that is desired, without entering the deeper (and muddier?) waters of metaphysics. Obviously, a reason must be given for doing that (and we shall try to provide some reasons why someone might do that in section 4), but restricting the scope of investigation like that is not unreasonable in principle.

Examples of ontological investigation in this specific sense are not difficult to find. One could ask whether there are numbers, for instance, or whether there are properties. As it is known, Bertrand Russell, in his brand of logicism (see Russell 1993), for instance, did not recognize classes as being part of the furniture of the world, but accepted properties and relations. Out of properties and relations, classes would be defined, and from them, natural numbers and, in fact, the rest of mathematics known at the time, was supposed to be obtained (there are difficulties, of course, with the axiom of infinity and axiom of reducibility, but let us not bother with that). Quine, on the other hand, adopting a version of physicalism with classes, had famously populated the world with bodies and classes; from classes the mathematicalia needed for science is introduced. Further examples will be discussed later.

When we so delimit the scope of ontology, so that it is understood as dealing specifically with what exists, metaontology also acquires a more specific aspect. The central issue is how to properly understand existence claims and their objectivity. On the nature of existence claims, to begin with that, one may dispute whether existence is properly captured by the existential quantifier, or perhaps whether an existence predicate is preferable, and also whether there is only one meaning for the existential quantifier or, yet, whether one may use distinct languages, each endowing the existential quantifier with a specific meaning (for doubts about the role of the existential 
quantifier as capturing what is real, see Azzouni 1998; for discussion on quantifier variance, see Hirsh 2009). On what concerns the objectivity of first-order ontological claims, the problem then becomes, as Chalmers (2009, p.77) puts it, to determine whether existence claims can be objectively true, or not. This leads to disputes between realists and antirealists about ontology. As we shall see, metaontology must have this specific aspect, because then it may be more clearly distinguished from issues discussed in metametaphysics.

\section{Metaphysics and metametaphysics}

Once ontology is confined to the specific task of providing the entities that populate the world and their respective types, what kind of role is left for metaphysics? As we have mentioned, following the distinction highlighted by Hofweber, metaphysics, in large measure, starts precisely where ontology stops, and develops from there. The idea is that part of the task of metaphysics consists of providing a description of the nature of the entities that we take as existing (and, in fact, metaphysics does even more, but some of the tasks attributed to metaphysics will not be dealt with here). But how is this supposed to work?

There are at least two possible approaches allowing us to plug a metaphysical layer over an ontology. We discuss them briefly here: 1) metaphysics provides a "metaphysical profile" or "metaphysical dressing" of the existing entities, or 2) metaphysics deals with questions of fundamentality, grounding, and dependence relations. Those two approaches are not necessarily excluding each other, but we shall merely mention one possible relationship between them here, without committing ourselves to a deeper discussion of the issue here (this is left for further work).

Let us begin with the claim that metaphysics is 
responsible for attributing a metaphysical profile. The idea is quite simple: once one admits that some kind $\mathrm{X}$ of entities exists, one may wish to go ahead and still provide for a "metaphysical dress" or a "metaphysical profile" for these entities (the idea is developed for a specific case in quantum mechanics in French 2013). That means that it is not enough to determine what there is and claim that one is committed to such entities; one must also be able to spell in detail what those entities are in metaphysical terms. Steven French (2014, p.48) puts it in terms of what he calls "Chakravartty's Challenge". Obviously, the terms of the challenge were advanced by Chakravartty himself:

One cannot fully appreciate what it might mean to be a realist until one has a clear picture of what one is being invited to be a realist about. (Chakravartty 2007, p.26)

In other words: when existence questions are connected with identifying the posits of our best scientific theories, so that one is being invited to believe in the existence of a certain kind of entity that a specific theory requires, then, according to this approach, one cannot simply say "I believe in the existence of such and such entities", without having a clear picture of what such entities are; having an ontology is not enough (according to French's version of Chakravartty's Challenge), one must "dress" such entities in clear metaphysical clothes. Notice: the point is that, on what concerns scientific realism, that deals precisely with the belief in the existence of the posits of our best scientific theories, the claim is that one cannot stop only at the ontological level, describing what the posits accepted are; one must provide for a metaphysical description of those posits to engender legitimate commitment (or, at least, that is how the description of the Challenge goes, and we shall not bring it 
into question here). It is instructive to check how French spells the details of how such an investigation might then proceed in a specific example. That is done just after French advances the above quote by Chakravartty:

But how do we obtain this clear picture? A simple answer would be, through physics which gives us a certain picture of the world as including particles, for example. But is this clear enough? Consider the further, but apparently obvious, question, are this particles individual objects, like chairs, tables, or people are? In answering this question, we need to supply, I maintain, or at least allude to, an appropriate metaphysics of individuality, and this exemplifies the general claim that in order to obtain Chakravartty's clear picture and hence obtain an appropriate realist understanding we need to provide an appropriate metaphysics. Those who reject any such need are either closet empiricists or 'ersatz' realists. (French 2014, p.48)

So, the idea is this: once physics (in this example) provides for particles (the ontology), one may ask a further question about the metaphysical nature of such particles. Provided that one takes the route of classifying the particles as particular objects, then, one of such additional metaphysical questions concerns the individuality of such particles, a question whose answer depends on an appropriate metaphysics of individuality; thence the need for a further layer of metaphysics over ontology (or so it is said, and again, we are here merely using the discussion of Chakravartty's Challenge as an example of how the connection between metaphysics and ontology may go, not discussing its merits). 
As we are discussing it, then, the idea that metaphysics concerns a profile for scientific ontology is very much on a par with issues on characterizations of scientific realism. The latter traditionally contains an existential aspect, e.g., the "belief in the ontology of scientific theories" (Chakravartty 2017). In this sense, one may be tempted to understand Chakravartty's Challenge as involving an exclusively existential demand: one can only be a realist about some entity provided that one assumes such entity exists. However, the existential aspect is not yet the clear picture demanded by French's version of "Chakravartty's Challenge", and this is made clear on several occasions: for instance, it would not count as legitimate realism a view "accepting that there are electrons, for example, but refusing to go any further and state what sorts of things electrons are" (French 2018a, p.395); and that is also confirmed by the claim that "when the object-oriented realist is unable to tell us whether electrons, qua objects, are individuals or not, it risks vacuity" (French 2015, p.194). Only those going beyond the ontological aspect of the entities that one is a realist about (but still assuming the existence of these very entities) and providing a metaphysical profile for such entities are 'legitimate' (i.e. non-ersatz) scientific realists. In this sense, ontology has chronological and methodological priority for debates concerning metaphysics and science in the context of scientific realism: in order for someone to assign a metaphysical profile to the entities of the ontology of a scientific theory, ontology needs to be on the table beforehand, even if only tentatively. This is not always the case in other debates on metaontology and metametaphysics (as we will see later in this paper), but it certainly is the case in this specific type of debate that we are interested in in this article - which is far from exhausting or reducing the extremely rich debate in these areas. It may also be argued that the metaphysical profile should be settled before one 
can even admit the entities in one's ontology. This might be the case for hardcore metaphysicians, who judge science from the standpoint of a first philosophy; but this is hardly the case in the metaphysics of science - where science must play at least a role in the methodological play.

Let us check with some more details how the relation of metaphysics and ontology, under this picture, is supposed to work. French, in the quote presented above, where he develops the formulation of Chakravartty's challenge, discusses the particular case of individuality. Recall: given an ontology of objects (particles), there is then a further issue (a metaphysical question) as to the metaphysical account of their individuality. In Lowe's (2003 p. 75) terms, the individuality question concerns what is it that makes an individual precisely that individual, and not any other individual, that is, the individuality of an item is granted by "whatever it is that makes it the single object that it is whatever it is that makes it one object, distinct from others, and the very object that it is as opposed to any other thing". Several approaches to individuality are available in the metaphysical literature; this is not the place to list and discuss them in details, but for the sake of illustration, let us just mention three of them and their main differences (again, see Lowe 2003 for a survey on individuality).

To begin with, some approaches to individuality attempt to grant individuality by means of a qualitative property that singles each item over every other, in such a way that numerically distinct items always correspond to qualitatively distinct items. A clear advantage of this approach is that whatever it is that confers the metaphysical feature of individuality to an item may also be what allows us to distinguish it from other items (an epistemic feature); the disadvantage of this approach is that qualitatively indistinguishable items are not allowed to be individuals. In order to account for the possibility of cases involving 
qualitative indiscernibility, an alternative approach to individuality proposes that individuality is conferred by an item of a non-qualitative nature, such as a Lockean substratum, or a haecceity. While a substratum is a self-individuated particular item that accounts for an entity's individuality by the simple fact of its presence in the item (it is an 'ingredient' of the individual), a haecceity is typically conceived as a nonqualitative property of being identical to a specific individual (for instance, Plato's haecceity would be the property 'to be identical with Plato'), so that it is instantiated only by one item. The main disadvantage of these approaches, of course, is that they leave the epistemology associated with the scientific posits (the particles, in French's example) miles away from the accompanying metaphysics; the item doing the metaphysical job (be it a substratum or a haecceity) is simply epistemically inaccessible. Another family of approaches to individuality attempt to attribute individuality by spatio-temporal trajectory; according to this approach, that which confers individuality to something is the fact that it has a trajectory exclusive for it (for a discussion on how these approaches fare in the case of quantum particles, see French and Krause 2006; for further details on the metaphysics of individuality, see Lowe 2003).

Without going into the details of the discussion, the general idea may be put as follows: given the entities provided by ontology, metaphysics adds a further layer of theoretical content by providing for further characterization on many distinct fronts. This is also called the toolbox of metaphysics approach, or the Viking approach to the metaphysics of science; given the ontology of a specific theory, the metaphysician of science goes to the metaphysics toolbox to find concepts that may be appropriated to dress the posits of science and provide for a clear version of realism (addressing, thus, Chakravartty's Challenge; see French 2018b). One may do the same kind of discussion for many 
items. Consider spacetime. From the ontology of General Relativity, let us say, one accepts that there is something called spacetime. As for the metaphysical questions, there is the dispute on the nature of spacetime and how spacetime relates with other entities, giving rise to such issues as the debate between tri-dimensionalism and fourdimensionalism, along with disputes involving endurantism and perdurantism. Note that these metaphysical views are all attempts to go into detail on the posit of ontology (spacetime).

For another case, consider properties. Once properties are adopted in the furniture of the world, there is the further issue of how to understand metaphysically the nature of properties. Some metaphysicians may say they are universals, others may think they are tropes. Those are different characterizations of properties, and they deal with issues distinct from the existence of properties.

This brings us to the second way metaphysics may build on ontology: discussions of grounding, - priority, fundamentality and dependence. Although leading to different discussions, all these terms refer to the same kind of basic intuition: the idea whether there is a sort of explanation that has a distinctively metaphysical nature, and as such, is investigated by metaphysics. The grounding relation is thought of as a suigeneris metaphysical explanatory relation, and may be employed either as building the less fundamental blocks of reality from the most fundamental ones (and there are disputes on which are the fundamental ones), or else, it may be used as a metaphysical tool to discuss such a grounding relation connecting non-fundamental relata, basically to explain the dependence relations between items that, again, need not be fundamental. While it is not our business to enter into a detailed discussion of what it means to provide for a metaphysical explanation, the idea is clearly a metaphysical thesis that could be seen as building 
on an ontology given beforehand. This would happen in cases where the grounding entities are of a given type, and may be seen as grounding entities of another type (and, again, the types of entities are provided by the ontology). For an instance that shall come to our attention very soon, consider the claim that properties may be considered as grounding the particulars, in a bundle theory, or whether the particulars are required to be there beforehand, and cannot be completely explained in terms of properties, or, perhaps, whether particulars could be explained, completely, in terms of relations (see, for instance, the discussion in French 2010, although French does not use the 'grounding' vocabulary, but rather 'dependence').

Given that this may also serve to distinguish metaphysics from ontology, it is interesting to check how Benovsky (2016) puts the point. According to him, the goal of metaphysics is to provide metaphysical explanations, so the goal of the metaphysician would be to find the primitive notions accounting for the behavior or function to be explained. The fundamental notions ground the nonfundamental ones. This, of course, goes a long way from finding the catalog of reality. According to Benovsky (2016, p.69),

The very point of building a metaphysical theory is to provide an explanation for some phenomena that we want to better understand (particularity of particulars, sharing the same property, persistence through time, ...) $[\ldots]$ the picture one gets of what metaphysics does and what it should do is thus not just to tell us what there is but, more importantly, $[\ldots]$ to discover what are the most fundamental notions, which are primitive and which are not. The idea here is that metaphysics does not and should not 
give us a list that is a sort of inventory of what there is, but rather a top-down structure of relations of 'grounding' or 'explanation' between types of entities or between concepts, saying which are primitive, which are not, and which are more fundamental than others.

While Benovsky does not use these claims to endorse the separation of labor between ontology and metaphysics (preferring to somehow defend that the real issue concerns fundamentality, against existence questions), we believe that this characterization fits pretty well with the distinction we have provided here, i.e. it does not exclude the possibility of complementary work between metaphysics, so conceived, and a distinct field of ontology whose concern is with existence questions.

To get a clearer idea of how this top-down structure may be based on ontology, consider the following example of a discussion by Koslicki (2016). According to Koslicki, one may agree on the question of whether there are tropes, but disagree on issues of fundamentality. One may assume (1) a pure trope theory according to which individuals are bundles of tropes, so that tropes are individuated primitively, or else (2) one may assume an impure trope theory according to which tropes and objects exist primitively, but in which tropes are individuated by the objects in which they occur (e.g. the redness of this ball, in opposition to the redness of that table). The main difference, then, does not concern whether tropes exist, but whether they have their individualities depending on the individuality of the objects they occur in. In the first theory, tropes are more fundamental than objects (they constitute the objects), so that objects cannot ground the tropes' individuality. In theory (2), tropes and objects are fundamental, but the individuality of the tropes is grounded in the individuality of 
the objects. Curiously, Koslicki uses the case to deny that ontology and metaphysics are the same thing, and to challenge the idea that metaphysics should deal with existence questions. That is, again, perfectly fine, and our distinction makes the case clearer. Assuming that there are important questions of fundamentality does not prevent one from also adopting a separation of work and recognizing that there is a field that deals specifically with existence questions. In fact, the distinction defended here dissolves disputes such as the one Koslicki and Benovsky are willing to substantiate, between questions of grounding and existence questions.

Koslicki (2016, p.233) concludes the following from this dispute:

Since the pure and the impure trope theorist, as we noted above, agree on the relevant existential questions, "Are there tropes?" and "Are there concrete particular objects?", their dispute cannot be characterized as a purely existential disagreement which would be amenable to a Carnapian or Quinean approach to ontology.

She then goes on to argue that metaphysics should not focus on existential questions. However, there needs not to be a dispute between metaphysics and ontology if each is given its due, as the distinction presently suggested does. Koslicki is right in claiming that this kind of dispute is out of the reach of the concerns of the Quinean and Carnapian approach to ontology, but mainly because Quine and Carnap were concerned with another family of problems, not because they thought existential problems should be the sole focus of metaphysics. Both approaches complement each other, and Koslicki is not right in trying to downplay the role of ontology in benefit of fundamentality questions. There is no 
issue of which should be the correct goal of metaphysics/ontology. Distinguishing both allows for a better characterization of each one's aims and methods.

Notice how this could map on the problem raised by French, concerning the individuality of particles: in the pure tropes approach, a particle is understood as a collection of tropes, and its objectuality is reduced to tropes only. Then, the ontology has only properties and relations, metaphysically dressed as tropes. On the impure picture, the ontology has objects, and properties and relations; the properties and relations are dressed as tropes, and one still has to provide an account for the individuality of the objects (through a substratum, haecceity, or even primitive individuality). But, again, the point is that both kinds of questions are not excluding each other, and one may turn to metaphysics to develop the discussion on the dependence relations. Notice that while the 'metaphysical dressing' perspective requires that we focus on providing for metaphysical descriptions of the posits, the grounding approach requires a focus on the relations between different metaphysical concepts. A clear relation between these, certainly, is possible, but we shall not discuss it here.

In fact, one of the defenders of the fundamentality of tropes (with no objects being fundamental), Campbell, as quoted by Koslicki, seems to be laboring on the very distinction advanced here. Campbell (1990) assumes that spacetime is a basic single trope, and that the other basic tropes are fields provided by physics; that is, once we have a physics-based ontology, we may provide for a metaphysical profile for them in terms of tropes:

All basic tropes are space-filling fields, each one of them distributes some quantity, in perhaps varying intensities, across all of spacetime. What are the plausible candidates for 
such cosmos-filling basic tropes? The ones that spring to mind first are those we already think of as having field characteristics, the fundamental forces recognized in contemporary physics. So we postulate superimposed fields for gravitation, electromagnetism, the weak and the strong nuclear forces. (Campbell 1990, p.146)

Again, ontology (as based on our best physics) populates the world with physical fields, while metaphysics deals with the profile of such fields and with priority questions (e.g. 'are there also fundamental objects?', 'can objects be grounded on tropes?' and so on). The picture described by Campbell makes better sense in the kind of distinction being advanced here, and seems to require a naturalistic approach to ontology (or at least a physics-based ontology), as we shall discuss soon. This also illustrates the kind of relationship that may obtain between both approaches to metaphysics under discussion here: a profile (in the sense of a required metaphysical profile to answer Chakravartty's Challenge) may be given in terms of the fundamental concepts, or in terms of concepts that are grounded in such fundamental concepts. Both approaches seem to be complementary in many interesting cases.

Before we move on, it is important to emphasize that the discussion of grounding relations is not necessarily connected to the discussion of fundamentality. Although one of the most basic concerns of grounding is clearly tied to the idea of building the world from the basics, in a unified metaphysical picture, so that the fundamental entities are the grounds for all the rest, one may clearly find other uses for grounding. A prominent case concerns employing the grounding relation for items that are not fundamental, in which case it may be thought of as describing a dependence 
relation (see Barnes 2012). What is important for us is that even in this case, one could see the problem of relating ontological types by a kind of metaphysical connection, without connecting the relata that account for the basis as fundamental in any sense. This is quite convenient for those willing to apply the tools of dependence relations to the entities posited by theories that are not considered basic (mostly, theories that are not from fundamental physics).

Once the distinction between metaphysics and ontology is properly made, a further question remains to be addressed, which is the kind of problem that metametaphysics would have to deal with in this kind of approach. Metametaphysics deals with the question of whether there are objective claims of metaphysics (whether claims of metaphysical profiles are truth-apt) and how to properly investigate such issues. This is not a question about quantifiers and appropriate representation of existence, or how to read ontological commitments from specific theories or pieces of discourse. Rather, they concern the role of the primitive or fundamental terms in a metaphysical picture and the proper understanding of grounding (for a nice discussion on this issue, see Benovsky 2016).

Now, the friends of grounding have already made it very clear that these questions are not the same questions of ontology, and so the problems in metametaphysics will have to be addressed differently from questions of metaontology. The idea that we may discuss the objectivity of quantification over universals or tropes makes little sense here. Rather, the discussion concerns whether tropes or universals are appropriate to ground whatever needs to be grounded, and how that is to be done. Notice that from this perspective the claim by Koslicki, quoted before, that on the debate between pure and impure trope theories one could assume that existence questions are settled, seems misguided: existence questions were supposedly settled because both sides accept 
an ontology of properties; also, from a metaphysical perspective, both sides accept that properties should be understood as tropes. The question of the existence of tropes is not on the same level as the question on the existence of properties.

This distinction impinges heavily on the relation between metaphysics and science on the one hand, and ontology and science, on the other. Certainly, this requires that the borders of each kind of investigation be reasonably made. But is this border possible to find?

In order to address this worry, we should consider a dilemma apparently faced by the proposal that we have been building up to now. ${ }^{2}$ It seems that the methodological division of labor we have been proposing between ontology and metaphysics is, to say the least, unfeasible. On the one hand, the argument could go, the distinction between ontology and metaphysics cannot be assumed to be rigid, i.e., both disciplines cannot be thought of as being independently carried out. The reason for that claim is that metaphysics seems to be required for the proper advancement of an ontology: all the relevant identity conditions and application conditions for our terms are provided by the previous involvement of metaphysics. Metaphysics provides identity conditions or individuality conditions for the entities, according to the 'no entity without identity' slogan. So, the argument would go, how can metaphysics come after ontology? Metaphysics is required beforehand, it seems.

This argument is quite interesting, but it relies on a confusion between two distinct senses of 'identity'. As Lowe 2015 has distinguished, 'identity' may mean at least two distinct things: the logical relation of numerical identity, or it may mean the individuality feature that confers to one entity its

2 The objection is a courtesy of a referee, to whom we are grateful for pointing this to us.

Manuscrito - Rev. Int. Fil. Campinas, v. 44, n. 2, pp. 01-51, Apr.Jun. 2021. 
individuality, being a property (in the sense that one could say that something 'has identity', meaning that it is an individual). Ontology may need that identity conditions or identity criteria are settled when 'identity' is understood in the first sense when one needs to count how many entities of a given kind are present and eventually distinguish them (and even this counting may be performed some times without the identity relation; see the discussion also in Lowe 2003). Counting and discussing how entities differ numerically is something that depends on the kind of terms that is being discussed, and need not be done through the metaphysical principle of individuality that confers each entity (if, at all) their individuality. It is enough that numerical difference is explained, in the sense of the logical identity. One can use properties, relations, spatial position, and many other features to account for numerical difference, but this does not mean that such things, properties, relations, spatial position, and others, are also being used to account for the metaphysical feature of individuality. This separation of tasks is important to be remarked because otherwise, counting would require a metaphysical principle of individuality in order to be effected; which is more than we can ask in most cases. What explains the individuality of something is most of the time not also that which accounts for the numerical difference in every situation. The question for identity, then, is ambiguous and may mean the mundane problem of numerical difference, or the more substantial, metaphysical question of individuality. The first need not be answered through metaphysics, the second requires metaphysics.

One could still complain, however. ${ }^{3}$ It could be added to the previous objection that debates appearing specifically in publications typically classified as metaphysical, such as the

${ }^{3}$ As a referee did, in fact. We owe to him/her the follow up to the original objection. 
debate on identity conditions for some specific kinds of entities $\mathrm{K}$, are, in fact, metaphysical tasks that must be performed before ontology can be addressed. Consider the particular case of 'person'. Typically, one could argue, traditional metaphysical debates in this case concern finding appropriate identity conditions (in the sense defined above) for persons, with the disputants offering two prominent choices, a psychological criterion, and a more bodilyoriented criterion:

(Psy) $x$ and y are the same person iff x's psychological make-up is appropriately psychologically related to y's psychological make-up (where appropriate psychological properties include memory, intentions, and so on).

(Bod) $x$ and $y$ are the same person iff x's body is the same as y's body.

So, the objection could go, it is metaphysics' task to determine such identity criteria, and that is done in order to grant discussions of ontological commitment with the required kind to get off the ground (still on the "no entity without identity' plan). As a result, metaphysics would be required for ontology.

But that is not a problem for our proposal. In fact, it concerns a sociological fact that discussions on ontology and metaphysics are typically made on the same kind of places, by the same kind of people. But that still does not prevent the more 'subject-matter' oriented distinction we are advancing here for methodological purposes. The fact that people working on the broad area of metaphysics do discuss identity criteria for some kinds does only mean that they are sometimes engaged in ontological debates too. In fact, the distinction we are advancing does not prevent one from seeing ontology as a subarea of metaphysics, as it is traditionally done (and as we do, see also the quote by Hofweber presented in the beginning). In this sense, one can say that people worried with identity criteria, in the sense 
proposed, are doing metaphysics, but in the quite uninteresting sense that everyone doing ontology is doing metaphysics. That is not enough to blur the subject matter distinction we are proposing.

Now, moving forward. Another evidence that such questions on identity criteria and individuality must be kept separated concerns disputes on non-individuality. One may have perfectly well-defined cardinality attributions (saying how many particles there are in a given context) while denying that individuality applies (see French and Krause 2006, chap.7, Lowe 2015). Also, the separation allows us to make a better sense of those theories that somehow conflate, on purpose, the two issues. Indeed, typically, bundle theories use distinguishability (a criterion used to establish logical identity and difference) as a principle of individuality (the metaphysical feature), and the fact that the same kind of feature plays both roles is used as an argument for the simplicity of the view, leading to its being preferable over competitors (or so it is said). However, notice, even this debate can only get off the ground if the distinction we are suggesting between the two senses of identity is preserved.

So, metaphysics is not really needed to get an ontology off the ground to begin with. But the argument against the distinction between ontology and metaphysics could also go from another route. It could be said that the boundary between metaphysics, ontology, and empirical science is furiy, and that then it seems hard to see in what sense we can read off our ontology from scientific theories without also reading a metaphysics, if it is admitted that such a scientific theory is itself informed by one's metaphysics. This claim could acquire some momentum from historical cases where scientific theories were indeed based on metaphysical views (like Descartes' physics, and Newton's views on space and time, influenced by his theological assumptions). Our distinction would end up being a-historical, and this would 
imply the failure in accounting for the fuzziness in the boundaries of metaphysics, ontology, and science.

Concerning the a-historicity point, we are abstracting the psychology of scientific creation, that is, the motivations that scientists have for proposing scientific theories (which can be, and often are, metaphysical). Our point is that whatever metaphysics it is, it cannot benefit from physics from an epistemological point of view. Certainly, one can use whatever one has in hands to create a theory; however, the success of the empirical theory does not transfer to that whatever motivation that gave rise to the theory. This also holds for theory choice. One may argue that some interpretations of non-relativistic quantum mechanics present a counter-example for this case inasmuch as metaphysical preferences come into play in the theoretical choices. Think about the poll presented by Schlosshauer (et al., 2013) in which working physicists expressed their preferences toward the interpretation of quantum mechanics and some interpretations were less popular than other ones particularly because of its relations with rather unpopular metaphysical views, such as the mind-body dualism in some versions of the Copenhaguen interpretation. A case in point of this preference is found in Lewis (2016, chap. 9) in which the so-called "Consciousness Causes Collapse" interpretation (due to mainly Wigner 1983, but see also de Barros and Oas 2017, and Bueno 2019) was ruled out of the space of possibilities for interpreting quantum phenomena because its relations with the mind-body problem. However, as we show in Arroyo and Arenhart (2019) show, this decision is not made on epistemic grounds but rather on a voluntarist basis. The epistemic warrant of a scientific theory does not bear on one's metaphysical preferences. There may be pragmatical, psychological, heuristic, and a whole spectrum of criteria that can orient one's preference in science, but this is not related to epistemic warrant. The same 
can be said about determinism and indeterminism: depending on one's metaphysical preference, one should prefer a deterministic theory, such as, say, Everettian quantum mechanics as opposed to other non-deterministic theorization of quantum phenomena. As far as we know, the decision process in the present state of art in the foundations of non-relativistic quantum mechanics is by large a voluntarist one (Dürr and Lazarovici 2020). So, in the same way, that science does not provide epistemic warrant to metaphysics, metaphysics does not provide privileged epistemic grounds for scientific theory choice.

Regarding the fuzzy border between disciplines, this case may be inevitable, as Berto and Plebani (2015, p.4) have anticipated, often occurring in situations such as the following: "authors A and B can seriously disagree on the metaphysical status of entities of kind F, which they nevertheless agree to include in the ontological catalogue" - which indicates that ontological debates often flow into metaphysical debates. Perhaps this serves to illustrate the fact that, bracketing problems, strategies and discussions between the two disciplines, we are not necessarily imposing barriers between them. Apparently, there are problems specific to each discipline, but this should not necessarily imply that there are no relationships between them. The point we would like to emphasize is that the epistemology of ontology is more linked to science than metaphysics. But this certainly does not prevent the connection of the disciplines.

It is worth noting that the distinction proposed here is not new, and has found fertile ground for debates in metaphilosophy even today. One of the examples that we can cite is Fine (2017, p.98), who distinguishes two ways of doing metaphysics: "one is with the nature of things, with what they are like; and the other is with reality, with what there is." The first is called "naive" or "pre-critical" metaphysics. According to this view, "[...] even if we adopt a 
negative ontological position on a certain class of objects, we can still sensibly pursue the question of what they are like" (p.100). As Fine (2017) presents it, the 'naive metaphysics' floats free even from ontology, not committing to the reality of the nature of the entities which are allowed to be investigated. The second task, on the other hand, would be linked to the description of how things are, in order to depend on an affirmative support (about what exists) of the ontology:

So, from a methodological point of view, each of the two branches of metaphysics is capable of informing the other. However, there remains a sense in which ontology is prior. For once we have established a negative answer to the ontological question, we cannot sensibly raise the question of what the objects of the given class are like. Metaphysics proper will, in this sense, presuppose a positive answer to the ontological question. (Fine 2017, p.99)

Again, the distinction proposed here is not made from scratch; it has been done over the past few years by other authors. The novelty of our proposal is that we are showing the fruitfulness of the distinction for science metaphysics, a sub-discipline of metaphysics in a broad sense. Thus, it is important to distance ourselves also from Fine for our purposes of the subarea: metaphysics of science. That is, the relationship with science is not peripheral, it is central to this paper. Therefore, even if, widely considered, metaphysics can in fact be conducted with a disciplinary independence in relation to ontology, to physics, or anything else, it is worth mentioning that the metaphysics of science is necessarily constrained to the ontology that comes, from somehow, of science - even though, as we have seen, it is a very small part of ontology. 


\section{Ontology, metaphysics, science}

The idea that the debate between Quine and Carnap on ontology somehow revived classical metaphysics, with Carnap's attempt to debunk metaphysics being defeated by Quine, is part of the analytic metaphysics folklore. Quine is seen as having provided not only for a revival of the discipline, but also for new methods for metaphysics; what is meant by that is the idea that the criterion of ontological commitment has shed light on how metaphysical questions could be profitably pursued. Of course, that emphasizes the role of quantifiers, and leads one to think of metaphysics as being concerned with existence questions. Also, that view leads to the recent revolt against the centrality of existence questions by part of the "grounding community" (see Schaffer 2009, Koslicki 2016). As we hope to have made clear in the previous section, prima facie there is room for everyone, provided that the proper distinctions are kept in mind. This has not been done so frequently.

For one case, consider Loux and Zimmerman (2003, p.2), who claim that due to Quine's approach to ontology, "philosophers no longer felt the need to conceal their interest in metaphysical issues, and there was something like a revival of traditional metaphysics". The diagnosis seems perfectly correct because traditional topics in metaphysics were indeed revived; however, the crucial question is whether such a revival was justified by Quinean lights. Thomasson (2016, p.122) puts the issue in similar terms when claiming that historically, it was thought that Carnap's approach to ontology was dead because Carnap's deflationism "was thought to have been defeated by Quine as he inaugurated a renaissance for serious metaphysics". Although Thomasson thinks that Quine failed in his criticism of Carnap, Quine is seen as opening the door for serious metaphysics.

This is not the place for a reconstruction of the Quine- 
Carnap debate (see Alspector-Kelly 2001 for a reassessment of the debate and its results). However, there are some lessons from this debate that may enlighten precisely why, as Koslicki has put it, their views may generate skepticism about metaphysics, but not about ontology, as in the sense that we propose in this paper. Also, it may shed some light on the methods of ontology and its relation to science (a topic that has concerned metaphysicians for some time now). This brief digression will substantiate our claim that a distinction between ontology and metaphysics, as defended here, in particular, may benefit the methodological discussions of those areas.

It is well known that Carnap advanced his latest approach to ontology in "Empiricism, Semantics, and Ontology" (1956). We shall not revise in detail Carnap's position here, which is well-known, but for the sake of having some points clear, we present the approach in a nutshell. Carnap suggests that to accept a new kind of entity, one must provide for a linguistic framework appropriate for that kind of entity. The framework must contain terms for referring to that kind of entity, rules of formation, and also rules for the adequate use of the language. Basically, that means that a framework must embody some kind of rules for the verification of its wellformed sentences. Systems are of a logical type if the rules indicate logical verification (deductions), and are of a factual type if those rules are empirical (verification by experience). The first kind of questions and answers are analytic, the second, synthetic. Inside the language framework, existence questions are formulated and answered using the resources of the system; these are the internal questions.

Examples of a framework of empirical kind concern the framework of things, such as rocks, kings, horses, sheets of paper, and so on. Inside a framework like this, one may formulate questions such as "do unicorns exist?" and "is there a sheet of paper over my table?", and verify empirically 
the answer to such questions; the answers are provided by experience. A logical framework may concern, for instance, the language of the arithmetic for natural numbers, which besides the terms for such entities, contains the rules of deduction to deal with those entities. Questions such as "are there numbers?", or "are there infinite twin prime numbers?" are formulated and answered inside this framework by logical means, so that the answers are all analytic.

Besides internal questions, there is also a second kind of question, the so-called external questions. These are questions about the admissibility of a framework. They are practical questions, concerning whether it is useful, for the purposes of science, to adopt a given language. So, one should not ask whether the number language corresponds to reality, or whether its adoption is justified in virtue of numbers really existing, but rather ask whether it is useful for scientific purposes to adopt such a language. Typical ontological questions raised by philosophers were thought to involve the justification of the admissibility of a language. Thus, the dispute between Platonists and nominalists in mathematics is seen as involving the justification of adopting a language quantifying over numbers, sets, and so on. Carnap does not think this will take us very far, because there is no way to decide the issue. It is rather a practical issue of the admissibility of a language, not a matter of truth or falsehood. This way of framing the point was considered as a deflation of the ontological problem, given that internal questions already assume the adoption of the language (hence, they are not the traditional philosophical question), while external questions are not theoretical, but rather practical. However, instead of a deflation of ontological questions, one could perhaps better consider it as a transformation of a philosophical problem, from a theoretical question into a practical issue. As Creath (2016, p.198) puts 
it:

It would be more accurate to say that Carnap wants to transform the discussion of philosophical ontology rather than to dismiss it. He wants to transform it from a theoretical dispute that has not gotten very far in a very long time (except for changes in fashion) into a practical issue over what tool to adopt. On Carnap's transformative approach we can productively investigate the practical consequences of using this or that tool and get on with the business of science without trying to find the one correct metaphysical position to adopt.

So, there is no issue of finding a correct metaphysical position to adopt, but rather the issue of whether adopting a given language is expedient for scientific purposes. There is not a question of the adequacy of a given language, only of its expediency. Of course, in the original Carnapian approach, a language must contain some rules of verification in order to cooperate with the verification theory of meaning, but what is relevant is that the external question, which is not theoretic, is judged by its expediency to deal with science.

This proposal by Carnap has close ties to the Quinean view on ontology. In his criticism of the Carnapian approach, Quine has advanced a few criticisms of the division between internal and external questions as related to the distinction between analytic and the synthetic. As it is now well studied (see again Alspector-Kelly 2001 for a clear exposition of the case), Quine seems to have misunderstood the doctrines of ESO, thinking that Carnap wanted somehow to safeguard ontological questions, just as logical 
and mathematical questions, as being analytic (without empirical content). He makes the issue of his disagreement with Carnap very clear in his (1966, p.134):

Within natural science there is a continuum of gradations, from the statements which report observations to those which reflect basic features say of quantum theory or of theory of relativity. [...] statements of ontology or even of mathematics and logic form a continuation of this continuum, a continuation which is perhaps yet more remote from observation than are the central principles of quantum theory or relativity. The differences here are in my view differences only of degree and not in kind. Science is a unified structure, and in principle it is the structure as a whole, and not its component statements on by on, that experience confirms or shows to be imperfect. Carnap maintains that ontological questions, and likewise questions of logical and mathematical principle, are questions not of fact but of choosing a convenient conceptual scheme or framework for science; and with this I agree only if the same be conceded for every scientific hypothesis.

That is, Quine took it that empirical hypotheses, on Carnap's view, were directly testable, while logic, ontology, and mathematics, involved issues related to the choice of a language. Questions in these fields — Quine thought — had analytic answers, by their own definition. Obviously, this is not the approach of Carnap in ESO, which sees logic and mathematical systems as on a par with empirical systems, although with distinct methods of verification. The point, for 
Carnap, as we have seen also with the quotation of Creath above, concerns the appropriate choice of a language for science, independently of whether that language deals with empirical or abstract entities.

Quine joins Carnap in treating ontological questions as a matter of the choice of a language. That is clear from his quotation above. However, the difference is that, while Carnap thought it possible to safeguard some epistemological privilege for science (empirical or logical) through the adoption of verification rules, Quine saw no reason to do that (due to his criticism of the analyticsynthetic separation). Science and ontology are in the same pragmatic boat, and the empirical hypotheses of science are also chosen in such a pragmatic fashion. They are not justified because of some direct verification, but due to their success in the system of science. The disagreement between Carnap and Quine, then, lies not on what concerns the proper understanding of ontology, but rather on what concerns the epistemology of science. Unlike Carnap, who conceived science as a subject matter satisfying the principle of verification, and hence, with epistemic privileges, Quine conceived of science as also indirectly related to experience, with hypotheses closer or farther from experience, depending on their place in the belief network. Just as the hypotheses of quantum mechanics help us to organize and predict future experience, so does ontology. We posit the existence of certain entities in our theories in order to systematize experience. That is how material bodies were introduced a long time ago in the course of human evolution, molecules, and more recently, curved spacetime and wave-like particles of quantum mechanics.

Basically, then, ontology, for both Quine and Carnap, is a matter of choosing a conceptual scheme for accommodating science. Notice that this involves accepting certain classes of entities as useful for science, and discarding others as not so useful. An ontology is justified in the 
measure of its success in systematizing science (Quine is known for adopting physicalism with classes, while Carnap was more open-minded on different conceptual schemes for accommodating science).

Given that brief summary, and that there is a pragmaticnaturalistic background common to Quine and Carnap, their approach to ontology suggests a method for ontology: if the items to which we will confer existence are those posited by our best scientific theories and those required to accommodate our best scientific theories, then one could try to extract ontological commitments from such theories. While those theories seem to come with an associated ontology, some issues remain to be discussed, as for instance, the precise delimitation of the ontology, the ontological commitments of the mathematics associated with the theory, and so on. However, what is relevant here is that there is a method for discussing these problems: the careful study of whether some of these postulated entities are necessary for the functioning of the corresponding scientific theory. Furthermore, constraints are imposed on such investigation just as they are imposed in the formulation of scientific theories: economy, fruitfulness, empirical adequacy, and other virtues expected from scientific theories are also to be employed to judge the appropriateness of ontology, given that they are part of the same naturalistic enterprise.

To bring the issue of naturalism even closer to ontology, current discussions in quantum mechanics may shed some light in the issue of choice of ontology. In fact, given that distinct interpretations of quantum mechanics populate the world with distinct entities and processes, choosing an interpretation is also, in part, choosing an ontology. As Ruetsche (2015, p.343) has argued, someone who believes realistically in quantum mechanics believes in an interpretation of quantum mechanics, which is roughly a description of how the world looks like according to 
quantum mechanics. That is why choosing an interpretation is, in part, choosing an ontology. Interpretations, in their turn, do end up having an impact on the practice of quantum mechanics, given that some of them even change the formalism of the theory. So, just as there are difficulties in currently settling an interpretation as the best, there are difficulties in settling an ontology as the most appropriate. But what is relevant is that the problem of choice of ontology is on the same footing as a scientific problem of setting an interpretation of quantum mechanics. It is not an external problem coming from outside of the scientific domain (that the problem of interpreting a theory is a scientific problem, see Sklar 2010). The fact that one cannot currently choose one interpretation over other rivals (and consequently, one ontology over its rivals) generates a well-known problem in the philosophy of science, the underdetermination of theory by evidence. But that is just a problem for those in the business of discovering which is the best account of reality.

At this point, perhaps the best strategy is to avoid focusing so much on issues of appropriate formalization, and focus more roughly on the more general lessons of a scientific theory for the ontology, even if this can only be considered as a preliminary step, to be further refined in latter stages. This would still be according to the general lines suggested by a broadly Quinean metaontology (and, for our purposes, this is illustrative; one could certainly also follow other approaches to ontology, for instance, in Meinongian lines, and attempt to check what are the existing entities a theory is positing too). For the purposes of this discussion, just remember that Quine (1966, p.125) would simply like to investigate, with the ontological commitment method, "[...] what, according to that theory, there is". In this sense, we are providing for the first steps to address the ontological problem. This is where we can call exemplification with quantum mechanics to gain clarity in the debate. For many 
authors in recent literature, providing a physical theory is providing an ontology and dynamics for the theory. We bring two examples of this. Esfeld (2019, p.222) states that a physical theory needs to:

(i) spell out an ontology of what there is in nature according to the theory, (ii) provide a dynamics for the elements of the ontology and (iii) deduce measurement outcome statistics from the ontology and dynamics by treating measurement interactions within the ontology and dynamics; in order to do so, the ontology and dynamics have to be linked with an appropriate probability measure. Thus, the question is: What is the law that describes the individual processes that occur in nature (dynamics) and what are the entities that make up these individual processes (ontology)?

In the same vein, Maudlin (2019, p.4, original emphasis) states that:

A physical theory should contain a physical ontology: What the theory postulates to exist as physically real. And it should also contain dynamics: laws (either deterministic or probabilistic) describing how these physically real entities behave. In a precise physical theory, both the ontology and the dynamics are represented in sharp mathematical terms.

In the case of non-relativistic quantum mechanics, what determines a quantum theory is the accepted solution to the measurement problem, e.g., the trilemma, due to Maudlin (1995), that emerges when it is conjointly assumed that i) the 
quantum description is complete, i.e. that nothing else describes the physical properties of the system of interest; ii) it admits only a linear dynamics, i.e., a dynamics that admits that a sum of results is a result; iii) provides unique measurement results, e.g., does not admit that a sum is a result. At least one of these items needs to be negated, and the negation of each of them results in a quantum theory with its own dynamics and ontologies. For example, denying the first results in a dynamic with hidden variables and an ontology of point particles (see Bohm and Hiley 2006); the negative of the second gives rise to a quantum theory with dynamics with collapse, and the ontology can vary from mass density to flashes (see Allori 2021); denying the third item, finally, results in a quantum theory with linear dynamics, some which postulate only the reality of the wave function (see Albert 2013), and others postulate the existence of multiverses (see Wallace 2012) but the point is that whatever the ontology, it is associated with a precise dynamic law and, therefore, linked to physics in some way. While we are not arguing for a full-blown defense of such a naturalistic method for ontology here, what we claim is that it should be clear that naturalism provides a way to keep the investigation of ontology closely tied to science, and also that there is a sign of a clearly delimited approach. Notice: in the specific case we are discussing here, ontology and entity postulation in science are on a par; the posits do play a role in the dynamics of physics, and even if we cannot decide which interpretation of quantum mechanics to choose merely on physical grounds, the problem is still a problem to be decided by physics.

Furthermore, and even more relevant for our purposes here, is the fact that this kind of approach does not transfer itself immediately to metaphysics. Notice that it is not fruitful for science to discuss whether the fields in physics are tropes or universals. This distinction (between tropes and universals) 
plays no role in the physical theory, neither in the dynamics, nor anywhere else. The same could be said of the notion of individuals and non-individuals in quantum mechanics. While some of the founders of quantum mechanics considered it appropriate - and even necessary - to discuss the metaphysical status of particles in quantum mechanics, they did so in popular science books in an attempt to convey the consequences of quantum theory to the general public, rather than just presenting it in their academic treatises and theoretical articles (see also French and Krause 2006, chap.3). This, we suspect, happens because these notions have no role to play in the theoretical development of physics. If they had a place in physics, they would be physics, not metaphysics!

Nota bene: this is not intended to mean that particular scientists may not have used some specific metaphysical conviction to address some scientific problem (see also our previous discussion concerning the menace of a-historicity of our proposal). Schrödinger (1998), to restrict ourselves to a telling case, seems to have suggested that quantum statistics and quantum non-individuality are closely associated, with quantum mechanics somehow requiring non-individuality if the new statistics (Bose-Einstein and Fermi-Dirac) are to make sense. However, as the latter analyses showed, this is not justified (see French and Krause 2006, chap.4); metaphysics does not play such a heavy role in physics, although it may be part of the heuristics and may guide the psychology of discovery in some cases. Our major point here is that such associated metaphysical pictures do not derive any epistemic privilege from this association with science. Basically, one can provide for the scientific explanation without using the metaphysical picture, and this is typically done (how could instrumentalists about quantum mechanics make sense of the theory in purely instrumental terms, if such heavy metaphysics were already involved in the most 
basic steps?).

As a result, naturalism has a (modest) place in ontology, it seems, but it is much harder to make sense of its role in metaphysics. In fact, seen as a question of fundamentality or metaphysical profile, metaphysics will most certainly be independent of science (see also Benovsky 2016 for a defense of this claim). The search for the most fundamental concepts may be understood as a search in physics, in the terms of the theory of everything, or as a fundamentality search, in the terms of metaphysics. In the first case, there will be an answer for the ontological question (what is there? Strings, fields, information, mathematical entities), but still not for the metaphysical question. In the second case, there will be an answer to the metaphysical question, but not for the ontological (physical) question. Metaphysics cannot directly benefit from the success of science in this case. Understood in terms of what we have called a 'pure metaphysics' in the previous section, metaphysics is an independent area of research.

Being detached from scientific practice, the answer to metaphysical questions will originate another kind of underdetermination, called the underdetermination of metaphysics by science (see also French 2014, chap.3). In fact, typical pure metaphysical theories are immune to science, because they just provide for answers to metaphysical questions that must be compatible with science (when applied to science), but cannot be derived from it. The metaphysical underdetermination, unlike the underdetermination of interpretation and ontology, cannot be expected to be resolved by further advances in science. In fact, given its level of abstraction, metaphysics must be floating free from science on a more general level.

An exception to this kind of metaphysical detachment from science concerns the more negative claims that some metaphysical theories may be incompatible with scientific 
theories (Arenhart 2012; Arroyo and Arenhart 2019). Some metaphysical theories may be plugged into some scientific theories and provide for accounts incompatible with scientific theories. A straightforward example concerns simple versions of bundle theory and quantum mechanics; according to a standard view, quantum entities cannot be seen as bundles of properties, given that they violate basic tenets of the view, such as the principle of the identity of indiscernibles (the PII, see French and Krause 2006, chap.4). Anyway, this negative approach does not allow us to endow metaphysical theories with positive evidence deriving from science, but only with negative evidence that a metaphysical theory fails in the attempts of applying certain metaphysical concepts to certain scientific theories. In other words: when applying a metaphysical theory to the ontology of science, one may have negative feedback due to incompatibility, but that is not enough to have any positive evidence for a metaphysical theory, and does not deal directly with the epistemology of pure metaphysics.

There are two distinct options that seem available once it is recognized that metaphysics may not enjoy any kind of epistemic privilege from science (be it pure or applied metaphysics). On the one hand, it is possible to follow the lead of Carnap and Quine and be anti-realist in metaontology, while being somehow realist about the ontology adopted (adoption of theory is a pragmatic matter, but from an internal perspective, the posited entities exist objectively). This is typically conjoined with skepticism about metaphysics, understood as the claim that one must go one step further and address deeper questions (i.e. Chakravartty's Challenge, or something to that effect). According to this approach, metaphysics goes just too far in asking for questions that are out of the epistemic reach of science, which, on the naturalist's eyes, constitute our best epistemology. On the other hand, it is possible to avoid 
metaphysical skepticism and think that the fundamental items are objective, they are a metaphysical dressing that should be compatible with whatever posits ontology may happen to advance. So, the view is compatible with distinct combinations of realism in metaontology and metaphilosophy. Obviously, the kind of views of metaphysics and ontology that fit with each option is different. This is a virtue of the distinction we have advanced, e.g. such second-order options are enlightened.

In case one is willing to embrace metaphysics as independent from science and having its own epistemology, then one will have to address the typical kind of issues that the current metametaphysics literature has already raised concerning the objectivity of metaphysical claims (and, quite importantly, the attacks following the manifesto by Ladyman and Ross 2007). To many philosophers, a close relationship with science would provide some relief in dealing with the endless uncertainties of metaphysics, endowing metaphysics with some epistemic justification from science (obviously, that kind of justification has not been achieved yet). Once metaphysics floats free from science, there is no hope for such direct relation, but perhaps that is just what metaphysicians (pure and applied) were expecting. We shall not judge such issues here.

\section{Conclusion}

What a proper distinction between the tasks of ontology and metaphysics opens up for us, then, is a clear scenario in which distinct sets of discussions may be conducted, with their own methods, problems, and each own with its resulting epistemology attached, which may be more or less connected to science. As we have argued, when ontology is restricted to problems of existence, problems of the first 
order and of the higher-order are subject to clearer examination. Perspectives for naturalizing ontology seem good, given that a pragmatic approach is adopted, following the lead of Carnap and Quine.

Also, it becomes clear that metaphysics deals with more general questions, which cannot benefit from a direct contact with science. In fact, differently from what happens in ontology, one cannot hope that fundamental entities (say tropes or universals, individuals or non-individuals) could intervene in scientific theorizing, or be part of it in a direct sense, as wave functions, quantum fields, or inertial forces, are. Things are different in ontology. The case of the interpretation of quantum mechanics makes the issue really clear. Consider many-worlds interpretations. They populate reality with worlds, so that our catalog of reality gets new entries with new worlds every time a measurement is made. Worlds, the posits of the interpretation, play a role in the economy of science. They are involved in theory choice and in theory development: the former happens because they allow a collapse-free formulation of quantum mechanics, therefore allowing retrodiction — and this could be considered an advantage over formulations of rivals which are not retroditional; the latter happens because it may be easy to provide for extensions to quantum field theory, easier than other interpretations such as Bohm's, and also provide for further scientific problems (e.g. accounting for probability in the many-worlds interpretation). However, no metaphysical profile of what worlds are (in metaphysical terms) does any such thing; the metaphysical dressing of worlds (if any such is available) does not impact on such problems. Then, metaphysical profiles are not directly involved in such theoretical constructs within science.

The door opens up then for two distinct attitudes: skepticism about metaphysics on the one hand, or adoption of metaphysics as an independent branch, with its own goals 
and methods, on the other. The current wave of naturalization of metaphysics is the result of the claim by philosophers who think that metaphysics by itself does not seem to have contributed to the objective knowledge of reality as science did (as defended by Ladyman and Ross 2007). The diagnosis was that metaphysics was simply way too far from the objective structure of reality, and should be closer to science, contributing to it, if it is to play any such role. However, the confusion between ontology and metaphysics has prevented real progress on this front. We hope our discussion has made it clear why such a relationship is a quite complex one.

Finally, for those willing to pursue metaphysical problems independently from science, the distinction provided here also brings some lessons. For example, instead of focusing on why the questions of existence are not the right kind of problem and underestimate the importance of this discussion, the focus should be on metaphysical questions and their epistemology. Once the problems addressed by metaphysics are clearly distinguished, the battle between the questions of existence on the one hand, and the questions of fundamentality and of the investigation of relations such as grounding and dependence on the other, is seen as ill-motivated.

Given all of this, maybe this is the place to address one further worry, and leave the paper with a promissory note. After all the discussion we have presented, it could be further objected that our separation of existence questions on one side (ontological), and fundamentality and grounding issues, on the other (metaphysics), puts some pressure on ourselves when it comes to a faithful account of important current debates; basically, the distinction fails to preserve the nature of important debates in metaphysics/ontology, which violate our separation of the fields. The fact is, it could be claimed, that some actual debates about existence concern whether we 
should quantify over fundamental entities only, or, over fundamental and non-fundamental entities as well (the Cameron/Fine debate in the literature). This requires that we apply the distinction between 'fundamental' and 'nonfundamental' to our inventory of reality, contrarily to the division of subject matter we are suggesting here. What then?

First off, as we already said, we do not think that the bracketing of the two disciplines by their respective tasks necessarily comes with a rigid barrier between them. Our main point here was to emphasize that the epistemology of ontological tasks is more linked to science than that of metaphysical tasks, and how methodological debates in the philosophy of science may benefit from that bracketing. Nevertheless, we believe that there is much to be discussed on this topic, due to ambiguities on the concepts employed, so that a full answer is simply not possible here. Space prevents us from a full treatment of the topic, but let us briefly deal with it. On the one hand, the fundamental/grounded dichotomy is in fact applied to ontology in the current use of the terminology. For instance, one finds claims to the effect that sets are fundamental, and numbers, say, are grounded on sets. Or, considering debates on ontic structural realism (see French 2014), we find claims that relations are fundamental, and objects are grounded in them. On the other hand, one could, following the illustration of Koslicki quoted in section 3 of this paper, apply the dichotomy in focus to what we are calling 'metaphysics'. In this latter sense, one can argue, for instance, that tropes are fundamental, while objects are grounded in them, or, alternatively, one could argue that both tropes and objects are fundamental. We have reserved the grounding/fundamental divide to metaphysics, and its application to metaphysical notions, as suggested, for instance, by Koslicki (not intended, it seems), and by Benovsky (2016) (intentionally, it seems). So, we have got at 
least half of it right. Did we get the other half wrong, when we prevented the dichotomy from applying to ontology?

Our proposal does in fact place the divide in terms at odds with what we find in current disputes, but we believe this is the right thing to do. When we allow the distinction to apply to the metaphysical debate, we capture such discussions as Koslicki and Benovsky already develop, allowing for a metaphysical kind of investigation that is independent of science. When we prevent that such a divide transpires to ontology, we are not abolishing the discussion on the scope of quantifiers, as it appears in the Cameron/Fine debate. Rather, we propose that this is not the same kind of dispute as it appears in metaphysics, as dealing with metaphysical concepts. If ontologists are to determine what is fundamental and what is grounded, as the current debate suggests, with the division applying to the problem of advancing catalog of reality, and as required by the current use of the terms, we end up in a situation where ontology and science are on the same business of finding the ultimate elements of reality (see also Bennett 2016), disputing on the same field. Worse yet: ontology would be claiming for itself the job of explaining (through a quite mysterious relation) how the furniture of the world is structured from fundamental to non-fundamental, from particles to tables composed of particles (or whatever one could take as fundamental in the required sense). This, again, would be an attempt to provide for an alternative to the already on-going scientific project of providing for such explanations, and ontology would, once more, be a rival to science. By restricting the mentioned divide to metaphysics, we allow it a subject matter not competing with science (since it needs not to find the ultimate posits of reality, but the dressing for the posits of science, and how such dressing structure themselves through grounding and dependence relations). When it comes to ontology, then, it would be 
better to use a different terminology for the related questions of when some entities are posited by a theory, and when they can be part of the furniture advanced by the theory, but still, be defined/reduced/explicated in terms of posits that are already available. This is clearly an issue in ontology of science, and depends on the workings of the scientific theory being dealt with, and on how one is going to understand ontological commitment. However the finer details are pursued, we believe, we can separate it from the issue of grounding/fundamentality, in order to avoid having to put ontology as an adversary to science. The debate on ontology, on whether to quantify over the fundamental entities, or over fundamental and everything else, may be converted on a debate on whether to quantify over the entities of the ultimate physical theory only, or on the entities of science more broadly, and, of course, on whether some kind of theoretical reduction exists for non-fundamental (now in the scientific sense) theories to fundamental theories (probably theories of physics). That is, the issue of quantifying over strings only, say, or quantifying over strings and tables and parrots, depends on what one takes to be the relations between fundamental physical theories and nonfundamental theories, and whether one takes science to be unified at all. These are delicate issues, and we can only point to them here. Recall: we are quite aware that this is not how terminology is in fact used in the debate, but we propose that we can make a better sense of the debate if things are appropriately separated.

\section{References}

Albert, D. 2013. Wave Function Realism. In: Ney, A. and Albert, D. (Eds.). The wave function: Essays on the metaphysics of quantum mechanics. Oxford: Oxford 
University Press, pp. 52-57.

Allori, V. 2021. Spontaneous Localization Theories with a Particle Ontology. In: Allori, V.; Bassi, A.; Dürr, D. and Zanghi, N. Do Wave Functions Jump? Perspectives of the Work of GianCarloGhirardi. Switzerland: Springer, pp. 73-94.

Alspector-Kelly, M. 2001. On Quine on Carnap on ontology. Philosophical Studies 102, pp.93-122.

Arenhart, J. R. B. 2012. Ontological Frameworks for Scientific Theories. Foundations of Science 17, pp.339356.

Arroyo, R. W., and Arenhart, J. R. B. 2019. Between physics and metaphysics: A discussion of the status of mind in quantum mechanics. In: de Barros, A. and Montemayor, C. (eds.) Quanta and Mind: Essays on the Connection between Quantum Mechanics and the Consciousness. Cham: Springer, pp.31-42.

Azzouni, J. 1998. On "On What There Is". Pacific Philosophical Quarterly 79, pp.1-18.

Barnes, E. 2012. Emergence and Fundamentality. Mind 121(484), pp.873-901.

Bennett, K. 2016. There is no special problem with metaphysics. Philosophical Studies 173, pp.21-37.

Benovsky, J. 2016. Metametaphysics. On Metaphysical Equivalence, Primitiveness, and Theory Choice. Switzerland: Springer.

Berto, F. and Plebani, M. 2015. Ontology and metaontology: a contemporary guide. London: Bloomsbury Publishing

Bohm, D. and Hiley, B. 2006. The Undivided Universe: An Ontological Interpretation of Quantum Theory. London: 
Routledge.

Bueno, O. 2019. Is There a Place for Consciousness in Quantum Mechanics?. In: de Barros, A. and Montemayor, C. (eds.) Quanta and Mind: Essays on the Connection between Quantum Mechanics and the Consciousness. Cham: Springer, pp.129-139.

Campbell, K. 1990. Abstract Particulars. Oxford: Blackwell.

Carnap, R. 1956. Empiricism, Semantics, and Ontology. In: Meaning and Necessity, pp.205-221. Chicago: The University of Chicago Press.

Chakravartty, A. 2007. A Metaphysics for Scientific realism. Knowing the Unobservable. Cambridge: Cambridge Un. Press.

- 2017. Scientific Realism. In: N. Zalta (ed.) The Stanford Encyclopedia of Philosophy. Metaphysics Research Lab, Stanford University.

Chalmers, D. 2009. Ontological Anti-Realism. In: D. Chalmers; D. Manley; R. Wasserman (eds.) Metametaphysics. New Essays in the Foundations of Ontology, pp.77-129. Oxford: Oxford Un. Press.

Dürr, D., and Lazarovici, D. 2020. Understanding Quantum Mechanics: The World According to Modern Quantum Foundations. Cham: Springer.

Creath, R. 2016. Carnap and ontology. Foreign travel and domestic understanding. In: S. Blatti, S. Lapointe (eds.) Ontology after Carnap, pp.190-199. Oxford: Oxford Un. Press.

de Barros, J. A., and Oas, G. 2017. Can We Falsify the Consciousness-Causes-Collapse Hypothesis in Quantum Mechanics?. Foundations of Physics 47, pp.1294-1308.

Manuscrito - Rev. Int. Fil. Campinas, v. 44, n. 2, pp. 01-51, Apr.-Jun. 2021. 
Esfeld, M. 2019. Individuality and the Account of Nonlocality: The Case for the Particle Ontology in Quantum Physics. In: Lombardi, O.; Fortin, S.; López, C. and Holik, F. (eds.) Quantum Worlds: Perspectives on the Ontology of Quantum Mechanics. Cambridge: Cambridge University Press, pp. 222244.

Fine, K. 2017. Naive Metaphysics. Philosophical Issues 27, pp. 98-113.

French, S. 2010. The interdependence of structure, objects, and dependence. Synthese 175, pp.177-197.

. 2013. Whither wave function realism? In: A Ney and

D. Z. Albert (eds.) Wave function realism, pp.76-90. Oxford: Oxford Un. Press.

- 2014. The structure of the world. Metaphysics and representation. Oxford: Oxford Un. Press.

2016. Response to my critics. Metascience, 25 (2), pp.189-196.

. 2018a. Realism and Metaphysics. In: J. Saatsi (ed.) The Routledge Handbook of Scientific Realism, pp.394-406. New York: Routledge. . 2018b. Toying with the Toolbox: how metaphysics can still make a contribution. Journal for General Philosophy of Science 49, pp.211-230.

French, S. and Krause, D. 2006. Identity in Physics. A historical, philosophical and formal analysis. Oxford: Oxford University Press.

Hirsh, E. 2009. Ontology and Alternative Languages. In: D. Chalmers; D. Manley; R. Wasserman (eds.) Metametaphysics. New Essays in the Foundations of Ontology, pp.231-259. Oxford: Oxford Un. Press.

Manuscrito - Rev. Int. Fil. Campinas, v. 44, n. 2, pp. 01-51, Apr.-Jun. 2021. 
Hofweber, T. 2016. Carnap's Big Idea. In: S. Blatti; S. Lapointe (eds.) Ontology After Carnap, pp.13-30. Oxford: Oxford Un. Press.

Koslicki, K. 2016. Questions of Ontology. In: S. Blatti; S. Lapointe (eds.) Ontology After Carnap, pp.220-241. Oxford: Oxford Un. Press.

Ladyman, J., and Ross, D. 2007. Everything Must Go: Metaphysics Naturalized. Oxford: Oxford Un. Press.

Lewis, P. 2016. Quantum Ontology. Oxford: Oxford Un. Press.

Loux, M. J., and Zimmerman, D. W. 2003. Introduction. In: M. J. Loux, and D. W. Zimmerman (eds.) The Oxford Handbook of Metaphysics, pp.1-7. Oxford: Oxford Un. Press.

Lowe, E. J. 2003. Individuation. In: Loux, M. J., Zimmerman, D. W., (eds.) The Oxford Handbook of Metaphysics. Oxford: Oxford Un. Press, pp.75-95. . 2015. Non-individuals. In: Guay, A., and Pradeu, T. (eds.) Individuals across the sciences. Oxford: Oxford Un. Press, pp.49-60.

Maudlin, T. 1995. Three measurement problems. Topoi 14.1, pp. $7-15$.

Maudlin, T. 2019. Philosophy of physics: Quantum theory. Princeton Foundations of Contemporary Philosophy. Princeton: Princeton University Press.

Quine, W. v. O. 1966. On Carnap's view on ontology. In: The ways of paradox and other essays, pp.126-134. New York: Random House.

Ruetsche, L. 2015. The shaky game +25 , or: on locavoracity. Synthese 192, pp.3425-3442.

Russell, B. 1993. Introduction to Mathematical Philosophy. New 
York: Dover Publications.

Schaffer, J. 2009. On what grounds what. In: D. Manley, D. Chalmers, R. Wasserman (eds.) Metametaphysics: New Essays on the Foundations of Ontology, pp.347-383. Oxford: Oxford Un. Press.

Schrödinger, E. 1998. What is an Elementary Particle? In Castellani, E. (ed.), Interpreting Bodies: Classical and Quantum Objects in Modern Physics. Princeton: Princeton University Press, 1998, pp.197-210.

Schlosshauer, M., Kofler, J., and Zeilinger, A. 2013. A snapshot of foundational attitudes toward quantum mechanics. Studies in History and Philosophy of Science Part B: Studies in History and Philosophy of Modern Physics, 44(3), pp.222-230.

Sklar, L. 2010. I'd love to be a naturalist - if only I knew what naturalism was. Philosophy of Science 77(5), pp.11211137.

Thomasson, A. 2016. Carnap and the Prospects for easy Ontology. In: S. Blatti; S. Lapointe (eds.) Ontology After Carnap, pp.122-144. Oxford: Oxford Un. Press.

Wallace, D. 2012. The emergent multiverse: Quantum theory according to the Everett interpretation. Oxford: Oxford University Press.

\section{$(c c)$ BY}

